\title{
Chronic urticaria in children:An update about etiology
}

\begin{abstract}
Urticaria, one of the most common dermatological disorders across the globe is characterized by itchy wheels with or without angioedema. The incidence of Urticaria in children is about $0.1 \%$ to $6 \%$ Chronic Urticaria is defined as a daily occurrence of spontaneous wheals, angioedema, or both for $>6$ weeks. The etiology of chronic Urticaria is multifactorial in children and it can be explored in $20 \%$ to $50 \%$ children. In most of children the cause of chronic Urticaria are either idiopathic or autoimmune.
\end{abstract}

Keywords: chronic urticaria, etiology, children
Volume 5 Issue 4 - 2017

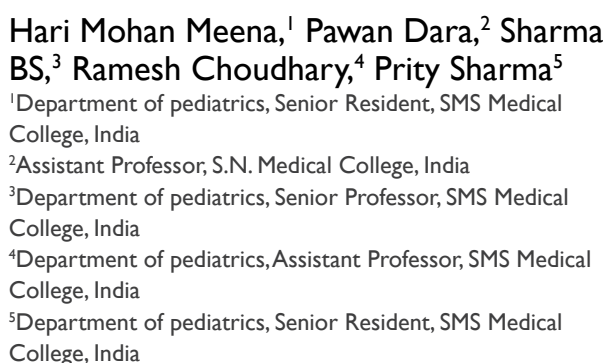

Correspondence: Hari Mohan Meena, Senior Resident, Department of pediatrics, Swai Man Singh Medical College, India, 302004, Email mohanmdped@gmail.com

\section{Introduction}

Chronic urticaria (CU) in children is a complex disease and the etiologies of chronic urticaria in children are somewhat different from adult. ${ }^{1}$ The prevalence of CU lasting $>6$ weeks is uncertain and varies among studies. The prevalence of chronic Urticaria in Spanish children is $18 \% .^{2}$ In Thailand $13 \%$ children were reported as having chronic urticaria. ${ }^{3}$ In another report, urticaria only was seen in $78.4 \%$, angioedema $6.6 \%$ and angioedema associated with urticaria observed in $15 \%$ of children with chronic urticaria. The chronic Urticaria is common in children of 6 to 11 year's age. ${ }^{4}$ There is no available information on the prevalence or differences in disease presentation according to age. There is no sex predisposition were found in children with chronic urticaria in recent studies unlike adults where $\mathrm{CU}$ is more common in female as compared to male. ${ }^{5}$ There are many etiological factors have been associated with chronic Urticaria in children but in most of cases are idiopathic. Infectious causes are more common in children than adult. ${ }^{6}$ This review is exploring the etiological agents of chronic Urticaria in children.

\section{Definition \& classification of chronic urticaria}

Chronic urticaria is defined as the occurrence of spontaneous wheals, angioedema or both for more than six weeks. Usually urticaria is classified on the basis of duration of presence of characteristic features and triggering agents. The 2014 revised European Academy of Allergy and Clinical Immunology (EAACI) guideline, classified the chronic urticaria into the two subgroups: chronic spontaneous urticaria and chronic inducible urticaria. ${ }^{7}$ Chronic spontaneous urticaria (CSU) is defined as the recurrent development of transient wheals (hives), angioedema (AE), or both for $>6$ weeks due to known or unknown causes. Previously this was known as chronic idiopathic urticaria. Infections, food intolerance, drugs and autoimmunity have been considered as cause of chronic spontaneous urticaria. ${ }^{7}$ Chronic inducible urticaria, earlier known as physical urticaria is characterized by presence of characteristic features of urticaria after triggering of specific stimulus. Chronic inducible urticaria often included dermographism, aquagenic, cholinergic, delayed pressure, solar, vibration, cold and heat urticaria as depicted in Table $1 .^{7}$

\section{Etiology of childhood chronic urticaria}

The etiology of childhood chronic urticaria is multifactorial and most commonformofchronicurticariaisspontaneousurticaria. Theetiologies of CU in children could be found out in $20 \%$ to $50 \%$ cases successfully. ${ }^{5}$ The following etiological factors are associated with chronic urticaria in children:

1. Chronic spontaneous urticaria: Most of studies have reported the wide variability of percentage of unknown cause of chronic urticaria in children. Volonakis et al. ${ }^{4} \mathrm{Had}$ not found out the cause of chronic urticaria in $75 \%$ children. In another study the most common cause of chronic urticaria was idiopathic. ${ }^{8}$

The following pathological condition included in causes of CSU.
I. Infections
II. Food allergy

III. Autoimmunity

IV. Drugs

2. Chronic inducible urticaria/physical urticaria: Almost identified causes are inducible urticaria, which cholinergic, symptomatic dermographism, cold, and pressure urticaria are most common forms.

\section{Infections}

Acute infections have also significant contributions in chronic urticaria. Recurrent respiratory and urinary infections have been associated with chronic urticaria. $^{9}$ Up to some extent Chlamydia and $\mathrm{H}$. helicobacter pylori are also play role in children with chronic 
urticaria observed that the infections in children are third most common etiology of chronic urticaria. ${ }^{10}$ Infection has been contributed about $7 \%$ to $13 \%$ as etiological factor of chronic urticaria in children. ${ }^{11}$

\section{Food allergy}

The role of food allergy in children with chronic urticaria is established up to some extent. Although, the correlation between

Table I Clinical classification of chronic urticaria

Types of chronic urticaria
Chronic spontaneous urticaria(CSU): Spontaneous appearance of
wheals, angioedema, or both $\geq 6$ weeks due to known or unknown causes

Chronic inducible urticaria(CIU): Triggered by specific Stimulus food allergy and acute urticaria is well known. Food additive and preservatives have role in severity of chronic urticaria. Volonakis et al were reported that $28 \%$ children have food allergy as cause of chronic urticaria. ${ }^{4}$. Several studies have demonstrated that about $10 \%$ to $12 \%$ of children with chronic urticaria have food allergy which is based on classical history, positive IgE test and skin prick test in some studies. ${ }^{8}$

\section{Trigger factor}

Stress, infection, drugs

Sub type of CIU

Symptomatic dermographism Minor trauma

Cold urticaria

Delayed pressure urticaria

Solar urticaria

Heat urticaria

Vibratory urticaria

Contact urticaria

Cholinergic urticaria

Aquatic urticaria
Swimming in cold water, cold wind

Sitting, lying, tight clothing

Sunshine

Hot bath/shower

Use of vibrating tools

Contact with offending agent

Exercise, emotion

Contact with hot or cold water

\section{Autoimmunity}

Autoimmunity can play role in chronic urticaria in children. An autologous serum skin test is usually performed to evaluate the etiology of chronic urticaria. Measurement of IgG auto antibodies can generally be done. However no correlation between IgG and ASST has been reported. The autologous serum skin test is positive in $35 \%$ to $50 \%$ children with chronic urticaria. ${ }^{12}$ Many autoimmune disorders including rheumatoid arthritis, systemic lupus Erythematosus, diabetes mellitus, inflammatory bowel disease, thyroid disease and celiac disease have been associated with chronic urticaria in children. ${ }^{12}$ Chronic urticaria was observed in $7 \%$ to $8 \%$ children with autoimmune thyroiditis. ${ }^{13}$ In another study, $4.3 \%$ of children with chronic urticaria had positive anti-thyroid antibody. ${ }^{14}$ If symptoms other than urticaria present, further evaluation must be done.

\section{Drugs}

Drugs usually cause acute urticaria rather than chronic urticaria in children. Nonsteroidal anti-inflammatory drugs (NSAIDs), penicillin and sulfonamide may cause chronic urticaria. One recent study found the NSAIDs and chronic urticaria in children and reported that $10 \%$ to $24 \%$ children with chronic urticaria have aspirin hypersensitivity. ${ }^{15}$

\section{Chronic inducible urticaria/Physical urticaria}

Physical urticaria often comprised dermographism, delayed pressure, solar, vibration and exercise and aquatic urticaria. Khakoo et al. had observed that among all type of physical urticaria, 38\% were dermographism, $19 \%$ aquagenic, $77 \%$ cholinergic, $17 \%$ combined them, $9 \%$ pressure, $9 \%$ heat, $2 \%$ hyperthermic and $4 \%$ idiopathic. ${ }^{16}$

\section{Cholinergic urticaria}

Cholinergic urticaria is caused by central hypothermia after hot water application, sweating. It is characterized by central small edema with large peripheral erythema with itching. Omalizumab can be used in treatment of chronic urticaria if conventional therapy is failed. ${ }^{17}$

\section{Conclusion}

Chronic urticaria in children has diverse etiology. Most of studies have reported that idiopathic urticaria is most common form of chronic urticaria in children. Apart from it, food allergy, autoimmunity, infections and drugs have crucial role in etiology of chronic urticaria in children.

\section{Contributions}

BSS got the idea about this review and help in making final draft, HMM write the manuscript, PD \& PS search the recent articles and collect the relevant information. RC help in final proof reading. HMM will act as guarantor.

\section{Acknowledgments}

Thank a lot to my wife Suman Meena for giving her valuable time to searching the recent published article..

\section{Conflicts of interest}

The authors declare no conflicts of interest.

\section{References}

1. Church MK, Weller K, Stock P, et al. Chronic spontaneous urticaria in children:itching for insight. Pediatr Allergy Immunol. 2011;22(1 Pt 1): $1-8$.

2. Ibanez MD, Garde JM. Allergy in patients under fourteen years of age in Alergológica 2005. J Investig Allergol Clin Immunol. 2009;19(Suppl 2):61-68.

3. Tuchinda M, Srimaruta N, Habanananda S, et al. Urticaria in Thai children. Asian Pac J Allergy Immunol. 1986;4(1):41-45.

4. Volonakis M, Katsarou-Katsari A, Stratigos J. Etiologic factors in childhood chronic urticaria. Ann Allergy. 1992;69(1):61-65.

5. Caffarelli C, Cuomo B, Cardinale F, et al. Aetiological factors associated with chronic urticaria in children:a systematic review. Acta Derm Venereol. 2013;93(3):268-272. 
6. Powell RJ, Du Toit GL, Siddique N, et al. BSACI guidelines for the management of chronic urticaria and angio-oedema. Clin Exp Allergy. 2007;37(5):631-650.

7. Zuberbier $\mathrm{T}$, Aberer W, Asero R, et al. The EAACI/GA(2) LEN/ EDF/WAO Guideline for the definition, classification, diagnosis, and management of urticaria: the 2013 revision and update. Allergy. 2014;69(7):868-887.

8. Jirapongsananuruk O, Pongpreuksa S, Sangacharoenkit $\mathrm{P}$, et al Identification of the etiologies of chronic urticaria in children:a prospective study of 94 patients. Pediatr Allergy Immunol. 2010;21(3):508-514.

9. Wedi B, Raap U, Wieczorek D, et al. Urticaria and infections. Allergy Asthma Clin Immunol. 2009;5(1):10.

10. Sackesen C, Sekerel BE, Orhan F, et al. The etiology of different forms of urticaria in childhood. Pediatr Dermatol. 2004;21(2):102-108.

11. Kauppinen K, Juntunen K, Lanki H. Urticaria in children. Retrospective evaluation and follow-up. Allergy. 1984;39(6):469-472.
12. Brunetti L, Francavilla R, Miniello VL, et al. High prevalence of autoimmune urticaria in children with chronic urticaria. $J$ Allergy Clin Immunol. 2004;114(4):922-927.

13. Dalal I, Levine A, Somekh E, et al. A Chronic urticaria in children:expanding the "autoimmune kaleidoscope". Pediatrics. 2000;106(5):1139-1141.

14. Levy Y, Segal N, Weintrob N, et al. Chronic urticaria:association with thyroid autoimmunity. Arch Dis Chil. 2003; 88(6):517-519.

15. Cavkaytar O, Arik Yilmaz E, Buyuktiryaki B, et al. Challenge-proven aspirin hypersensitivity in children with chronic spontaneous urticaria. Allergy. 2015;70(2):153-160.

16. Khakoo G, Sofianou-Katsoulis A, Perkin MR, et al. Clinical features and natural history of physical urticaria in children. Pediatr Allergy Immunol. 2008;19(4):363-366.

17. Metz M, Bergmann P, Zuberbier T, et al. Successful treatment of cholinergic urticaria with antiimmunoglobulin E therapy. Allergy. 2008;63(2):247-249. 